Academic Platform Journal of Engineering and Science

\title{
Yol Gözlemleyicisi Kullanarak Parametrik Belirsizlik İçeren MR Damperli Süspansiyon Sisteminin Yarı Aktif Doğrusal Olmayan Uyarlamalı Kontrolü
}

\author{
${ }^{1}$ Mahmut Paksoy, ${ }^{2}$ Gökhan Kararsız, ${ }^{* 3}$ Muzaffer Metin \\ ${ }^{1}$ Yıldız Teknik Üniversitesi, Makine Müh. Bölümü, İstanbul, Türkiye, mpaksoy@ yildiz.edu.tr, \\ ${ }^{2}$ Yıldız Teknik Üniversitesi, Makine Müh. Bölümü, İstanbul, Türkiye, kararsiz@yildiz.edu.tr, \\ *3YYıldız Teknik Üniversitesi, Makine Müh. Bölümü, İstanbul, Türkiye, mmetin@yildiz.edu.tr, \\ Araştırma Makalesi \\ Geliş Tarihi: 24.06.2019 \\ Kabul Tarihi: 23.08.2019
}

$\ddot{O} z$

Taşıt titreşimlerini bastırmak amacıyla yapılan kontrolör tasarımlarında yol girişinin ölçülmesi zor ve ek sensörler gerektirdiği için maliyetlidir. Bu yüzden, manyetoreolojik damper ile donatılmış süspansiyon sistemine sahip çeyrek taşıt modeline ait düşey titreşimleri bastırmak için yol bozucusunun önceden bilinmesine veya ölçülmesine ihtiyaç duymayan bir lineer olamayan uyarlamalı kontrolör tasarımı bu çalışma ile yapılmıştır. Bu amaç doğrultusunda, yolun farklı frekans, farklı genlik ve farklı faz değerlerine sahip bilinmeyen ve birbirinden farklı sinüzoidallerin toplamından oluştuğu varsayımı yapılarak Lyapunov esasına dayalı bir yol gözlemleyicisi tasarlanmıştır. Ele alınan kontrolör, model içerisinde yer alabilecek parametrik belirsizliklere karşı da en iyi cevabı garanti edecek şekilde tasarlanmıştır. Önerilen yöntemin etkinliği pasif cevaplar ile zaman ve frekans alanında karşılaştırılarak incelenmiştir.

Anahtar Kelimeler: Taşıt titreşimi, manyetoreolojik damper, uyarlamalı kontrol, parametre belirsizliği, yol gözlemleyicisi

\section{Semi-Active Nonlinear Adaptive Control of MR Damper Suspension System which Contains Parametric Uncertainty using Road Observer}

\author{
${ }^{1}$ Mahmut Paksoy, ${ }^{2}$ Gökhan Kararsız, ${ }^{* 3}$ Muzaffer Metin \\ ${ }^{1}$ Yildiz Technical University, Mechanical Eng. Dept., Istanbul, Turkey, mpaksoy@ yildiz.edu.tr \\ ${ }^{2}$ Yildiz Technical University, Mechanical Eng. Dept., Istanbul, Turkey, kararsiz@yildiz.edu.tr \\ *3 Yildiz Technical University, Mechanical Eng. Dept., Istanbul, Turkey, mmetin@yildiz.edu.tr
}

\begin{abstract}
In designed controllers in order to suppress the vehicle vibrations, the road input measurement process is difficult and costly as additional sensors are required. Therefore, a nonlinear adaptive semi-active controller is designed without road disturbance measurement for quarter vehicle model with suspension system equipped with magnetorheological damper in this study. For this aim, a road observer based on the Lyapunov approach is designed by assuming that the road consists of a sum of different frequency, different amplitude, and different phases. The controller is designed to guarantee the best response to the parametric uncertainties that may be present in the model. Effectiveness of the suggested method is investigated in time and frequency domain with using numeric simulations and some graphics.
\end{abstract}

Keywords: Vehicle vibrations, magnetorheological damper, adaptive control, parametric uncertainties, road observer

\section{GíRiş}

Taşıtlarda süspansiyon sisteminin görevi yoldan kaynaklanan titreşimleri bastırmak ve iyi bir yol tutuşu sağlamaktır. Bunun için pasif, aktif ve yarı aktif olmak üzere üç tip süspansiyon sistemi kullanılır. Bunlardan aktif süspansiyon sistemi yüksek enerjiye ihtiyaç duyar ve pahalı bileşenlere sahiptir. Pasif süspansiyon sistemi maliyeti düşük olması sebebiyle en çok tercih edilen süspansiyon sistemidir, fakat titreşim sönümleme performansı limitlidir. Bu yüzden araştırmacılar son yıllarda az enerjiye ihtiyaç duyan ve aktif sistemlere göre daha ucuz maliyete sahip ve pasif sisteme 
göre daha iyi titreşim sönümleme performansı sunan yarı aktif süspansiyon sistemlerine yönelmişlerdir. Bunlardan en çok tercih edileni ise manyetoreolojik (MR) damperdir. MR damperin titreşim sönümleme performansı ile ilgili son yıllarda birçok çalışma yapılmıştır. Bu çalışmalar, sadece nümerik simulasyonlar [1], laboratuvar ortamında oluşturulan deneysel sistemler [2], gerçek taşıtlar [3] ya da simülasyon çevriminde donanım yönteminin kullanıldığı hibrit yöntemlerle yapılmıştır [4].

MR damperin en önemli özelliği uygulanan manyetik alan şiddetine bağlı olarak viskozitesinin değişebilmesidir. MR damper genel olarak piston, MR sıvısı, akümülatör ve manyetik sargıdan oluşur. MR sönümleyicinin fiziksel modeli Şekil 1'de verilmiştir. MR damper içerisindeki MR sıvısı taşıyıcı sıvı içerisine mikron seviyede manyetik parçacıklar eklenerek elde edilir. Taşıyıcı sıvı olarak genelde su, silikon bazlı yağ veya petrol bazlı yağ kullanılır. Pistonun ucundaki boşlukta aynı zamanda elektromıknatıslar bulunmaktadır. $\mathrm{Bu}$ elektromıknatıslar manyetik alan oluşturmazsa MR sıvısı normal akışkan özelliği gösterir. Fakat, elektromıknatıslar manyetik alan oluşturduğunda MR sıvısını boşluktan geçerken içinde bulunan manyetik parçacıklar sıralı bir şekilde dizilerek sıvının viskozitesini değiştirir ve olduğundan daha viskoz bir sıvıymış gibi davranmasını sağlar. Bu da MR sönümleyicinin manyetik alan şiddetini ayarlayarak kontrol edilmesini ve böylece MR sönümleyicinin oluşturduğu sönüm kuvvetinin değişmesini sağlar. Bunun için gerekli güç miktarı oldukça düşük olup bir akü ile bile sağlanabilir. Ayrıca herhangi bir sebepten dolayı güç kesilirse MR sönümleyicinin pasif sönümleyici özelliği göstermesi, emniyet açısından önemlidir.

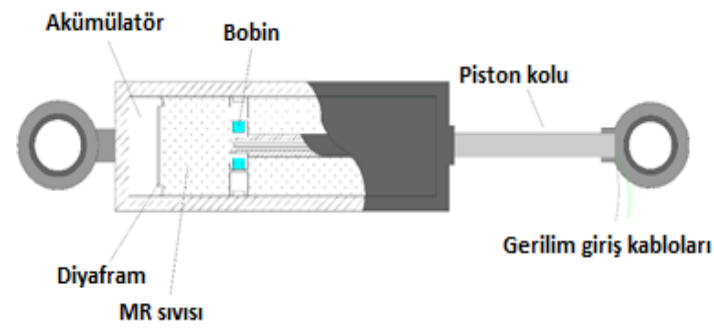

Şekil 1. MR damperin genel yapısı [5].

$\mathrm{Bu}$ çalışmada çeyrek taşıt modeli kullanılarak, MR damper kullanılan bir süspansiyon sistemine sahip binek aracın yol kaynaklı titreşimleri, doğrusal olmayan uyarlamalı bir kontrolörle bastırılmıştır. Kontrolör tasarımında yol düzensizliğinin ölçümüne ihtiyaç duyulmadan gözlemleyici tasarımı ile tahmin edilmesi ve modele ait bazı parametrelerin belirsiz kabul edilmesi çalışmanın orijinal yönlerini ortaya koymaktadır. Önerilen kontrolörün başarısı ISO C sınıfı bir yol girişine karşılık elde edilen simülasyon sonuçları ile ortaya konmuştur.

\section{MR DAMPER MATEMATÍKSEL MODELI}

MR damper lineer olmayan histerezis karaktere sahiptir. Dolayısıyla, matematiksel olarak modellenmesi zordur.
Fakat, literatürde MR damperin dinamiğini gerçeğe yakın şekilde yansitabilecek parametrik ve parametrik olmayan modeller bulunmaktadır. Bunlardan parametrik olmayan modellere Chebyshev polinomları [6], yapay sinir ağları [7], yapay sinir ağları ve bulanık mantığın bir arada kullanıldığı ve "neuro-fuzzy" olarak isimlendirilen yöntem [8] örnek olarak gösterilebilir. Fakat parametrik olmayan modellerin dezavantajı, çok fazla deney ile veri toplama işlemi gerektirmesi, bunun da işlem ve data yükünü çok fazla arttırmasıdır. Parametrik modeller ise, sürtünme prensibine dayal1 Dahl, Lugre, Bouc-Wen, Bingham ve Bouc-Wen modelinin biraz daha geliştirilmesi ile elde edilen Modifiye Bouc Wen modelleridir.

Bu çalışmada, MR damper matematiksel modeli kontrolör denklemlerinde kullanılacaktır. Çeyrek taşıt modelinde parametrik belirsizliklerin de olduğu düşünüldüğünde oldukça karmaşık denklemler ortaya çıkabilir. Örneğin Modifiye Bouc-Wen modeli gerçeğe çok yakın sonuçlar verebilmesine karşın, karmaşık yapısıyla kontrolör tasarımında işlemleri çok zorlaştıracaktır. Dolayısıyla, hem gerçeğe yakın hem de basit matematiksel yapıya sahip bir model bu çalışma için daha uygun olacaktır. Bu yüzden Lugre modeli bu çalışmada tercih edilmiştir. Lugre modeline ait denklemler denklem (1) ve denklem (2)'deki gibidir [9].

$$
\begin{aligned}
& f_{m r}=\sigma_{a} z+\sigma_{0} z v+\sigma_{1} \dot{z}+\sigma_{2} \dot{x}+\sigma_{b} \dot{x} v \\
& \dot{z}=\dot{x}-a_{0}|\dot{x}| z
\end{aligned}
$$

Burada; $f_{m r}$ MR damper sönüm kuvveti, $\dot{x}$ MR damperin rölatif hızı ve $v$ ise MR dampere uygulanan gerilimdir. $f_{m r}$ sönüm kuvveti uygulanan gerilime bağlı olarak değiştiği için $v$, aynı zamanda kontrol girişidir. $z$ MR damperin iç dinamiğini tanımlamada kullanılan iç değişken, $\sigma_{0}$, v'ye bağlı olarak değişen z'nin rijitliği, $\sigma_{1}$, z'nin sönüm katsayısı, $\sigma_{2}$, viskoz sönüm katsayısı, $\sigma_{a}$, z’nin rijitliği, $\sigma_{b}, v^{\prime}$ ye bağlı olarak değişen viskoz sönüm katsayısı ve $a_{0}$ ise sabit bir katsayıdır. Lugre modeline ait parametreler Tablo 1'de verilmiştir.

Tablo 1. Lugre model parameterleri [10]

\begin{tabular}{|c|c|c|c|}
\hline Prm. & Değer & Prm. & Değer \\
\hline$\sigma_{a}$ & $76000(\mathrm{~N} / \mathrm{m})$ & $\sigma_{2}$ & $1153.3(\mathrm{Ns} / \mathrm{m})$ \\
$\sigma_{0}$ & $320000(\mathrm{~N} / \mathrm{mV})$ & $\sigma_{b}$ & $315(\mathrm{Ns} / \mathrm{mV})$ \\
$\sigma_{l}$ & $3.21(\mathrm{Ns} / \mathrm{m})$ & $a_{0}$ & $1400(1 / \mathrm{m})$ \\
\hline
\end{tabular}

Lugre modeli MATLAB-Simulik programı yardımıyla oluşturulmuş ve $5 \mathrm{~mm}$ genlikli $2 \mathrm{~Hz}$ frekansına sahip bir rölatif yer değiştirme MR damperin ucuna uygulanarak 0,1 ve 2 volt gerilimler için kuvvet miktarının voltaja göre değişimi (histerisiz karakteri) Şekil 2'de incelenmiştir. 


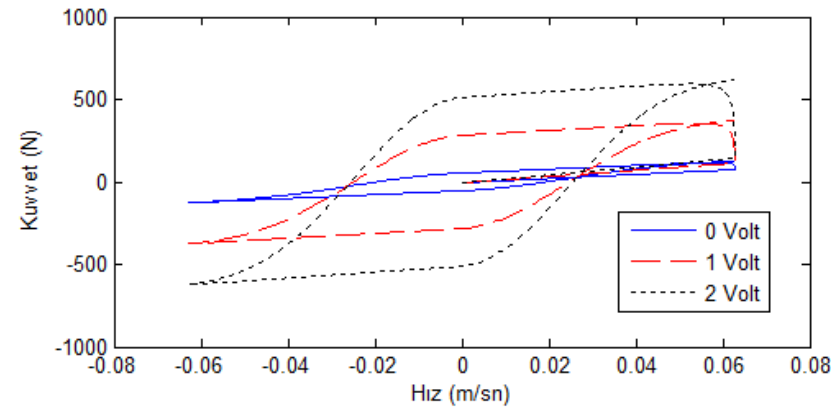

Şekil 2. MR damperin histerezis karakteri.

\section{CEYREK TASSIT MODELİ}

Çeyrek taşıı modeli $x_{1}$ ve $x_{2}$ ile tanımlanan iki serbestlik derecesine sahiptir. Basit yapısı nedeniyle akademik çalışmalarda çokça tercih edilir. Modelde asılı olan $\left(\mathrm{m}_{1}\right)$ ve asılı olmayan $\left(\mathrm{m}_{2}\right)$ olmak üzere iki kütle vardır. Bu kütleler süspansiyon sistemi vasıtasıyla birbirine bağlanırlar. Süspansiyon yayı, katsayısı $k$ olan lineer bir yay olarak modellenebilir. Burada, tekerleğin sönümü çok küçük olduğundan dolayı ihmal edilerek, yay katsayısı $k_{t}$ olan lineer bir yay olarak modellenebilir. $d$ ifadesi çeyrek taşıt modeline uygulanan yol girişini temsil etmektedir. Çeyrek taşıt fiziksel modeli Şekil 3 'te görülmektedir. Modele ait hareket denklemleri ise denklem (3) ve denklem (4)'teki gibi elde edilebilir.

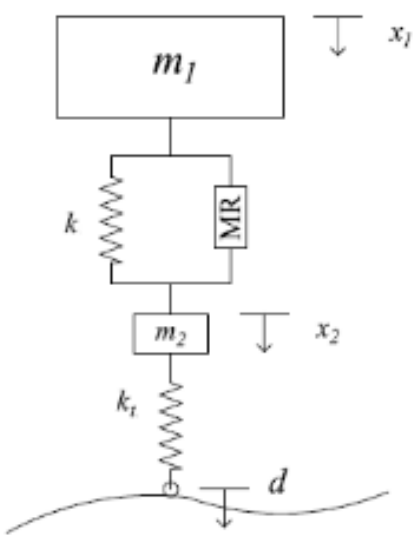

Şekil 3. Çeyrek taşıt modeli.

$$
\begin{aligned}
& m_{1} \ddot{x}_{1}+k\left(x_{1}-x_{2}\right)+f=0 \\
& m_{2} \ddot{x}_{2}-k\left(x_{1}-x_{2}\right)+k_{t}\left(x_{2}-d\right)-f=0
\end{aligned}
$$

Denklmelerde $f$ Lugre modeli yardımıyla hesaplanan MR damper sönüm kuvvetini ifade etmektedir. Çeyrek taşıt modeline ait parametre değerleri Tablo 2'de verilmiştir.

Tablo 2. Çeyrek taşıt model parameterleri [11]

\begin{tabular}{|c|c|c|c|}
\hline Parametre & Değer & Parametre & Değer \\
\hline$m_{l}$ & $338.5 \mathrm{~kg}$ & $k$ & $15000 \mathrm{~N} / \mathrm{m}$ \\
$m_{2}$ & $59 \mathrm{~kg}$ & $k_{t}$ & $190000 \mathrm{~N} / \mathrm{m}$ \\
\hline
\end{tabular}

\section{UYARLAMALI TASARIMI \\ (ADAPTIF) \\ KONTROLÖR}

Lineer olmayan uyarlamalı kontrolcü tasarımında aşağıdaki kabuller yapılmıştır;

Kabul 1: Çeyrek taşıt modelinde $m_{2}$ kütlesi tekerlek ve aks kütlesidir ve değerlerinin çok fazla değiş̧mediği bilinmektedir. Bu yüzden $m_{2}$ kütlesinin değerlerinin bilindiği kabul edilmiştir. Çeyrek taşıta ait diğer parametrelerin bilinmediği ve $x_{1}, x_{2}$ yer değiștirme ve hızlarının ölçüldü̈̆ü kabul edilmiştir.

Kabul 2: Lugre matematiksel modeline ait $a_{0}$ hariç diğger parametrelerin bilinmediği kabul edilmişstir.

Kabul 3: d yol girişlerinin ölçülemediği ve yol girişlerinin farkl frekans, genlik ve faz farkina sahip sinüzoidallerin toplamından oluştuğu kabul edilmiştir.

\subsection{Yol Gözlemleyici Tasarımı}

Yol gözlemleyicisi tasarımında, yol girişinin Kabul 3'e göre sinüzoidal bir sinyal olduğu söylenebilir ve dolayısıyla denklem (5)'teki gibi ifade edilebilir;

$$
\frac{k_{t}}{m_{2}} d(t)=\sum_{i=1}^{q} A_{i} \sin \left(w_{i}+\emptyset_{i}\right)
$$

Burada $q$ frekans sayısı, $A_{i}$ genlik, $w_{i}$ frekans $\emptyset_{i}$ ise faz olarak tanımlanmıştır. $q$ hariç diğer parametrelerin bilinmediği kabulü yapılmıştır. Burada $q$ değeri maksimum baskın frekans miktarı olarak seçilmelidir [12]. Bu çalışmada çeyrek taşıt modelinin iki serbestlik dereceli olduğu ve iki adet doğal frekansa sahip olduğu göz önünde bulundurularak $q=2$ olarak seçilmiştir. Bu durumda yol bozucu girişi aşağıdaki şekilde modellenebilir [13, 14];

$$
\begin{aligned}
& \dot{P}=S P \\
& \frac{k_{t}}{m_{2}} d(t)=h^{t} P
\end{aligned}
$$

Burada; $P \in \Re^{2 q}, S$ ise yol bozcusunun frekansına bağlı matristir. Bu durumda yol bozucu girişi [14]'e göre parametrize edilebilir. $G \in \mathfrak{R}^{2 q \times 2 q}$ gözlemlenebilir, $S$ ve $G$ 'nin spektrumları ayrık olduğundan, Sylvester denkleminin bir çözümü $\left(N \in \Re^{2 q \times 2 q}\right)$ olduğunu dikkate alarak denklem (8) yazılabilir [13];

$$
N S-G N=l h^{t}
$$

$Z_{r}=N P$ koordinat transformasyonu yapıldığında denklem (9) ve denklem (10) aşağıdaki formda yazılabilir. 


$$
\begin{aligned}
& \dot{z}_{r}=G z_{r}+l\left(\frac{k_{t}}{m_{2}} d(t)\right) \\
& \frac{k_{t}}{m_{2}} d(t)=z_{r}^{T} \theta
\end{aligned}
$$

Burada $\theta^{T}=h^{T} N^{-1} \in \Re^{2 q x 1}$ bilinmeyen katsayı matrisidir. Zamana bağlı bilinmeyen vektör olan $Z_{r 1}(t)$ 'i tahmin etmek için kavramsal bir gözlemleyici tasarlanmıştır. Bu durumda bilinmeyen yol giriși denklem (11)'deki gibi önerilmiștir [4, 12-14];

$$
z_{r}^{T} \theta=\delta^{T} \theta+\zeta^{T} \theta+\varphi_{1}^{T} \beta_{1}+\varphi_{2}^{T} \beta_{2}
$$

Burada, $\zeta, \varphi_{1}$ ve $\varphi_{2}$ ifadeleri denklem (12)-(15)'teki gibi tanımlanmıştır.

$$
\begin{aligned}
& \zeta=\varphi+l \dot{x}_{2} \\
& \dot{\varphi}=G\left(\varphi+l \dot{x}_{2}\right)-\frac{l}{m_{2}} f \\
& \dot{\varphi}_{1}=G \varphi_{1}-l\left(x_{1}-x_{2}\right) \\
& \dot{\varphi}_{2}=G \varphi_{2}-l x_{2}
\end{aligned}
$$

Burada; $\beta_{1}=\theta \frac{k}{m_{2}}, \beta_{2}=\theta \frac{k_{t}}{m_{2}}$ dir.

\subsection{Uyarlamalı Kontrolör Tasarımı}

Çeyrek taşıt modelinin hareket denklemleri, denklem (3) ve denklem (4)'te verilmiştir. Bu denklemler matris formunda aşağıdaki gibi yazılabilir;

$$
M \ddot{x}_{s}+K_{s} x_{s}+H f+L\left(\frac{k_{t}}{m_{2}} d\right)=0
$$

Denklem (2) ifadesi denklem (1)'in içine yazılırsa Lugre modeli denklem (17)'deki hali alacaktır;

$$
f=\sigma_{a} z+\sigma_{0} z v+\sigma_{1} \dot{x}-\sigma_{1} a_{0}|\dot{x}| z+\sigma_{2} \dot{x}+\sigma_{b} \dot{x} v
$$

Daha kompakt formda ise denklem (18)'deki gibi yazılabilir;

$$
f=\rho_{1} \theta_{1}+\rho_{2} \theta_{2}
$$

Burada $\rho_{2}$ ifadesi bilinen (ölçülebilen), $\rho_{1}, \theta_{1}$ ve $\theta_{2}$ ifadeleri ise bilinmediği varsayılan ifadelerdir ve dolayısıyla tahmin edilmeleri gerekmektedir. $\mathrm{Bu}$ yüzden tahmin ifadesi denklem (19)'daki gibi yazılabilir;

$$
\hat{f}=\widehat{\rho_{1}} \widehat{\theta_{1}}+\rho_{2} \widehat{\theta_{2}}
$$

Bu çalışmada tahmin hatası gösterimi genel olarak $\tilde{*}=*-\hat{*}$ şeklindedir. Burada “ $\widetilde{*}$ ” gösterimi tahmin hatasını, “ $\widehat{*}$ ” gösterimi ise tahmin edilen değeri ifade etmek için kullanılmıştır.

Çeyrek taşıt modelinde titreşimleri bastırabilmek için istenen ideal durum asılı olan ve asılı olmayan kütlelerin yer değişim değerlerinin sıfır olmasıdır. Bu yüzden hata ifadesi denklem (20)'deki gibi tanımlanabilir;

$$
e=x_{s}-x_{d}
$$

Burada, $x_{d}$ olması arzu edilen yer değiştirme değeridir. Yer değişimlerin değerlerinin sıfir olması istendiği için $x_{d}=0$ olmalidir. Bu durumda $e=x_{s}$ olacaktır. Burada, $x_{s}=$ $\left[\begin{array}{ll}x_{1} & x_{2}\end{array}\right]^{T}$ şeklinde tanımlanmıştır. MR damper kuvveti hıza bağlı olarak değiştiği için hızları da hata ifadesine eklemek kontrolcünün başarısını arttırabilir. Bu amaçla hata dinamiği olarak $r$ fonksiyonu denklem (21)'deki gibi tanımlanmıştır;

$$
r=\lambda_{a} \dot{e}+\lambda_{b} e
$$

Burada, $\lambda_{a}, \lambda_{b}$ katsayı matrisleridir. Hata dinamiği sağdan kütle matrisi ile çarpılıp denklem (16)'da verilen ifade yerine yazılırsa denklem (22) elde edilir;

$$
\begin{gathered}
M \dot{r}=M \lambda_{b} \dot{x}_{s}-\lambda_{a} K_{s} x_{s}-\lambda_{a} L \delta^{T} \theta-\lambda_{a} L \zeta^{T} \theta \\
-\lambda_{a} L \varphi_{1}{ }^{T} \beta_{1}-\lambda_{a} L \varphi_{2}{ }^{T} \beta_{2} \\
-\lambda_{a} H f \\
M \lambda_{b} \dot{x}_{s}-\lambda_{a} K_{s} x_{s}=Y \phi
\end{gathered}
$$

Burada bilinen ve bilinmeyen parametreler bir arada matris biçiminde yazılır ve denkleme $\lambda_{a} H \hat{f}$ ifadesi eklenip çıkarılırsa denklem (23) elde edilir;

$$
\begin{aligned}
M \dot{r}=Y \phi-L_{a} \delta^{T} & \theta-L_{a} \zeta^{T} \theta-L_{a} \varphi_{1}{ }^{T} \beta_{1} \\
& -L_{a} \varphi_{2}{ }^{T} \beta_{2}-\Xi f+\Xi \hat{f}-\Xi \hat{f}
\end{aligned}
$$

Burada, $\Xi=\lambda_{a} H$ ve $\lambda_{a} L=L_{a}$ olarak tanımlanmıştır. $Y$ ve $\phi$ matrisleri ise denklem (24) ve denklem (25)'teki gibidir;

$$
\begin{aligned}
& \left.Y=\left[\begin{array}{cccc}
\lambda_{b 1} \dot{x}_{1} & 0 & \left(-\lambda_{a 1} x_{1}+\lambda_{a 2} x_{2}\right) & 0 \\
0 & \lambda_{b 2} \dot{x}_{2} & \left(\lambda_{a 2} x_{1}-\lambda_{a 2} x_{2}\right) & -\lambda_{a 2} x_{2}
\end{array}\right]\right] \\
& \phi=\left[\begin{array}{llll}
m_{1} & m_{2} & k & k_{t}
\end{array}\right]^{T}
\end{aligned}
$$

Kontrolcünün amacı titreşimleri bastırmak için uygun gerilim miktarını belirlemektir. Bu yüzden, denklem (23)'te gerilime bağlı olan ifadeleri bir araya toplamak için $X$ ve $u_{x}$ ifadeleri denklem (26) ve denklem (27)'deki gibi tanımlanabilir;

$$
\begin{aligned}
& X=-\hat{\theta}_{11} \hat{z}+\hat{\theta}_{13}|\dot{x}| \hat{z}-\hat{\theta}_{21} \dot{x} \\
& u_{x}=\left(\hat{\theta}_{12} \hat{z}+\hat{\theta}_{22} \dot{x}\right) v
\end{aligned}
$$


$X$ ve $u_{x}$ ifadeleri denklem (23)'te yerine koyulursa denklem (28) elde edilir;

$$
\begin{aligned}
M \dot{r}=Y \phi-L_{a} \delta^{T} & \theta-L_{a} \zeta^{T} \theta-L_{a} \varphi_{1}{ }^{T} \beta_{1} \\
& -L_{a} \varphi_{2}{ }^{T} \beta_{2}+\Xi X-\Xi u_{x} \\
& -\Xi \rho_{2} \tilde{\theta}_{2} \\
& -\Xi\left[\theta_{11} z-\hat{\theta}_{11} \hat{z}+\theta_{12} v z\right. \\
& \left.-\hat{\theta}_{12} v \hat{z}-\theta_{13}|\dot{x}| z+\hat{\theta}_{13}|\dot{x}| \hat{z}\right]
\end{aligned}
$$

Denklem (28) incelendiğinde $\Xi u_{x}$ ifadesi denklem (29)'daki gibi kontrol sinyali olarak seçilebilir;

$$
\begin{aligned}
\Xi u_{x}=K r+Y \hat{\phi} & -L_{a} \zeta^{T} \hat{\theta}-L_{a} \varphi_{1}{ }^{T} \hat{\beta}_{1}-L_{a} \varphi_{2}{ }^{T} \hat{\beta}_{2} \\
& +\Xi X \\
& +\Xi\left(-\hat{\theta}_{11} \xi_{1}-\hat{\theta}_{12} \xi_{2} v\right. \\
& \left.+\hat{\theta}_{13}|\dot{x}| \xi_{3}\right)
\end{aligned}
$$

$\mathrm{Bu}$ durumda, MR dampere uygulanması gereken gerilim denklem (30)'daki gibi elde edilebilir;

$$
\Xi v=\frac{\left(\begin{array}{c}
K r+Y \hat{\phi}-L_{a} \zeta^{T} \hat{\theta}-L_{a} \varphi_{1}{ }^{T} \hat{\beta}_{1}- \\
L_{a} \varphi_{2}{ }^{T} \hat{\beta}_{2}+\Xi X+\Xi\left(-\hat{\theta}_{11} \xi_{1}+\hat{\theta}_{13}|\dot{x}| \xi_{3}\right)
\end{array}\right)}{\left(\hat{\theta}_{12} \hat{z}+\hat{\theta}_{22} \dot{x}+\hat{\theta}_{12} \xi_{2}\right)}
$$

Denklem (29)'da verilen kontrol sinyali ifadesi denklem (28)'de yerine yazılır ve elde edilen denkleme (31) eklenip çıkarılırsa kapalı çevrim sistemin denklemi (32)'deki gibi elde edilir.

$$
\begin{aligned}
\Xi\left[ \pm \theta_{11}\left(\hat{z}+\xi_{1}\right) \pm \theta_{12}\right. & \left.v\left(\hat{z}+\xi_{2}\right)+\hat{\theta}_{13}|\dot{x}|\left(\hat{z}+\xi_{3}\right)\right] \\
M \dot{r}=Y \tilde{\phi}-K r & -\Xi \rho_{2} \tilde{\theta}_{2}-L_{a} \delta^{T} \theta-L_{a} \zeta^{T} \tilde{\theta} \\
& -L_{a} \varphi_{1}{ }^{T} \tilde{\beta}_{1}-L_{a} \varphi_{2}{ }^{T} \tilde{\beta}_{2} \\
& +\Xi\left[-\tilde{\theta}_{11}\left(\hat{z}+\xi_{1}\right)\right. \\
& \left.-\theta_{11}\left(\tilde{z}-\xi_{1}\right)\right] \\
& +\Xi\left[-\tilde{\theta}_{12} v\left(\hat{z}+\xi_{2}\right)\right. \\
& \left.-\theta_{12} v\left(\tilde{z}-\xi_{2}\right)\right] \\
& +\Xi\left[\tilde{\theta}_{13}|\dot{x}|\left(\hat{z}+\xi_{3}\right)\right. \\
& \left.+\theta_{13}|\dot{x}|\left(\tilde{z}-\xi_{3}\right)\right]
\end{aligned}
$$

$z$ ifadesi iç değişkendir ve fiziksel olarak ölçülmesi mümkün değildir dolayısıyla gözlemleyici tasarlanması gereklidir. $z$ için tasarlanan gözlemleyici denklem (33)'teki gibidir;

$$
(\dot{\tilde{z}})=-a_{0}|\dot{x}| \tilde{z}
$$

\subsection{Kararlıık Analizi}

Kararlılık analizi için bir aday pozitif tanımlı Lyapunov fonksiyonu seçilmelidir. $\mathrm{Bu}$ çalışmada aday Lyapunov fonksiyonu " $V$ " denklem (34)'teki gibi seçilmiştir;

$$
\begin{aligned}
V=\frac{1}{2}\left[r^{\mathrm{T}} M r+\tilde{z}^{2}\right. & +\tilde{\phi}^{T} \Gamma_{\phi}{ }^{-1} \tilde{\phi}+\widetilde{\theta}^{T} \Gamma_{\theta}{ }^{-1} \tilde{\theta} \\
& +\tilde{\beta}_{1}{ }^{T} \Gamma_{\beta_{1}}^{-1} \tilde{\beta}_{1}+\tilde{\beta}_{2}{ }^{T} \Gamma_{\beta_{2}}^{-1} \tilde{\beta}_{2} \\
& +\varepsilon \delta^{T} P_{\delta} \delta+\tilde{\theta}_{2}{ }^{T} \Gamma_{2}^{-1} \tilde{\theta}_{2} \\
& +\frac{1}{\gamma_{1}} \tilde{\theta}_{11}^{2}+\frac{1}{\gamma_{2}} \tilde{\theta}_{12}^{2}+\frac{1}{\gamma_{3}} \tilde{\theta}_{13}^{2} \\
& +\theta_{11}\left(\tilde{z}-\xi_{1}\right)^{2} \\
& \left.+\theta_{12}\left(\tilde{z}-\xi_{2}\right)^{2}+\theta_{13}\left(\tilde{z}-\xi_{3}\right)^{2}\right]
\end{aligned}
$$

Denklem (34)'ün zamana göre türevi alınırsa denklem (35) aşağıdaki gibi elde edilir;

$$
\begin{aligned}
\dot{V}=r^{T} M \dot{r}+\tilde{z} \dot{\tilde{z}} & +\tilde{\phi}^{T} \Gamma_{\phi}{ }^{-1} \dot{\tilde{\phi}}+\widetilde{\theta}^{T} \Gamma_{\theta}{ }^{-1} \dot{\tilde{\theta}} \\
& +\tilde{\beta}_{1}{ }^{T} \Gamma_{\beta_{1}}^{-1} \dot{\tilde{\beta}}_{1}+\tilde{\beta}_{2}{ }^{T} \Gamma_{\beta_{2}}^{-1} \dot{\tilde{\beta}}_{2} \\
& -\varepsilon \delta^{T} \delta+\tilde{\theta}_{2}{ }^{T} \Gamma_{2}^{-1} \dot{\tilde{\theta}}_{2} \\
& +\frac{1}{\gamma_{1}} \tilde{\theta}_{11} \dot{\tilde{\theta}}_{11}+\frac{1}{\gamma_{2}} \tilde{\theta}_{12} \dot{\tilde{\theta}}_{12} \\
& +\frac{1}{\gamma_{3}} \tilde{\theta}_{13} \dot{\tilde{\theta}}_{13}+\Theta
\end{aligned}
$$

Denklem (35)'te karmaşıklığı önlemek amacıyla $\Theta$ ifadesi denklem (36)'daki gibi tanımlanmıştır;

$$
\begin{aligned}
\Theta=\theta_{11}\left(\tilde{z}-\xi_{1}\right) & \left(\dot{\tilde{z}}-\dot{\xi}_{1}\right) \\
& +\theta_{12}\left(\tilde{z}-\xi_{2}\right)\left(\dot{\tilde{z}}-\dot{\xi}_{2}\right)+\theta_{13}(\tilde{z} \\
& \left.-\xi_{3}\right)\left(\dot{\tilde{z}}-\dot{\xi}_{3}\right)
\end{aligned}
$$

Denklem (32)'de tanımlanan kapalı çevrim sistem denklemleri denklem (35)'te yerine yazılırsa, denklem (37) aşağıdaki gibi elde edilir; 


$$
\begin{aligned}
\dot{V}=r^{T}\{Y \tilde{\phi}-K r & -\Xi \rho_{2} \tilde{\theta}_{2}-L_{a} \delta^{T} \theta-L_{a} \zeta^{T} \tilde{\theta} \\
& -L_{a} \varphi_{1}{ }^{T} \tilde{\beta}_{1}-L_{a} \varphi_{2}{ }^{T} \tilde{\beta}_{2} \\
& +\Xi\left[-\tilde{\theta}_{11}\left(\hat{z}+\xi_{1}\right)\right. \\
& \left.-\theta_{11}\left(\tilde{z}-\xi_{1}\right)\right] \\
& +\Xi\left[-\tilde{\theta}_{12} v\left(\hat{z}+\xi_{2}\right)\right. \\
& \left.-\theta_{12} v\left(\tilde{z}-\xi_{2}\right)\right] \\
& +\Xi\left[\tilde{\theta}_{13}|\dot{x}|\left(\hat{z}+\xi_{3}\right)\right. \\
& \left.\left.+\theta_{13}|\dot{x}|\left(\tilde{z}-\xi_{3}\right)\right]\right\} \\
& +\tilde{z}\left(-a_{0}|\dot{x}| \tilde{z}\right)+\tilde{\phi}^{T} \Gamma_{\phi}{ }^{-1} \dot{\tilde{\phi}} \\
& +\widetilde{\theta}^{T} \Gamma_{\theta}{ }^{-1} \dot{\tilde{\theta}}^{2} \tilde{\beta}_{1}{ }^{T} \Gamma_{\beta_{1}}^{-1} \dot{\tilde{\beta}}_{1} \\
& +\tilde{\beta}_{2}{ }^{T} \Gamma_{\beta_{2}}^{-1} \dot{\tilde{\beta}_{2}}-\varepsilon \delta^{T} \delta \\
& +\tilde{\theta}_{2}{ }^{T} \Gamma_{2}^{-1} \dot{\tilde{\theta}}_{2}+\frac{1}{\gamma_{1}} \tilde{\theta}_{11} \dot{\tilde{\theta}}_{11} \\
& +\frac{1}{\gamma_{2}} \tilde{\theta}_{12} \dot{\tilde{\theta}}{ }_{12}+\frac{1}{\gamma_{3}} \tilde{\theta}_{13} \dot{\tilde{\theta}}_{13}+\Theta
\end{aligned}
$$

$\dot{\tilde{\phi}}=-\dot{\hat{\phi}}=-\Gamma_{\phi} Y^{T} r, \quad \dot{\tilde{\theta}}=-\dot{\hat{\theta}}=\Gamma_{\theta} \zeta L_{a}^{T} r$

$\dot{\tilde{\theta}}_{2}=-\dot{\hat{\theta}}_{2}=\Gamma_{2} \rho_{2}{ }^{T} \Xi^{T} r$

$\dot{\tilde{\beta}}_{1}=-\dot{\hat{\beta}}_{1}=\Gamma_{\beta_{1}} \varphi_{1} L_{a}^{T} r, \quad \dot{\tilde{\beta}}_{2}=-\dot{\hat{\beta}}_{2}=\Gamma_{\beta_{2}} \varphi_{2} L_{a}^{T} r$

$\dot{\tilde{\theta}}_{11}=-\dot{\hat{\theta}}_{11}=\gamma_{1}\left(\hat{z}+\xi_{1}\right) \Xi^{T} r$

$\dot{\tilde{\theta}}_{12}=-\dot{\hat{\theta}}_{12}=\gamma_{2} v\left(\hat{z}+\xi_{2}\right) \Xi^{T} r$

$\dot{\tilde{\theta}}_{13}=-\dot{\hat{\theta}}_{13}=-\gamma_{3}|\dot{x}|\left(\hat{z}+\xi_{3}\right) \Xi^{T} r$

$\dot{\xi}_{1}=-a_{0}|\dot{x}| \xi_{1}-\Xi^{T} r, \quad \dot{\xi}_{2}=-a_{0}|\dot{x}| \xi_{2}-v \Xi^{T} r$

$\dot{\xi}_{3}=-a_{0}|\dot{x}| \xi_{3}-|\dot{x}| \Xi^{T} r$

Denklem (38)'de verilen güncelleme kuralları ve yardımcı filtre ifadeleri denklem (37)'de yerine yazilırsa ve $r^{T} L_{a} \delta^{T} \theta$ ifadesi için Young's eşitsizliği uygulanırsa Lyapunov fonksiyonun zamana göre türevi denklem (39)'daki gibi elde edilir;

$$
\begin{aligned}
\dot{V}=-r^{T} K_{I} r-\Upsilon & \delta^{T} \delta-a_{0}|\dot{x}| \tilde{z}^{2} \\
& -\theta_{11} a_{0}|\dot{x}|\left(\tilde{z}-\xi_{1}\right)^{2} \\
& -\theta_{12} a_{0}|\dot{x}|\left(\tilde{z}-\xi_{2}\right)^{2} \\
& -\theta_{13} a_{0}|\dot{x}|\left(\tilde{z}-\xi_{3}\right)^{2}
\end{aligned}
$$

Yukarıdaki denklem incelendiğinde $K_{I}$ ve $\Upsilon$ pozitif seçilirse $\dot{V}$ ifadesi her zaman negatif olacaktır. Dolayısıyla sistem
LaSalle-Yoshizawa teoremine göre kararlıdır.

\section{SİMÜLASYON BULGULARI}

Önerilen yöntemin etkinliğini incelemek amacıyla MATLAB-Simulink programı ile bazı simülasyonlar yapılmıştır. Süspansiyon sisteminde stroğun aşılmaması gerekliliği ve yolcu konforundaki iyileşme beklentisi nedenleriyle, süspansiyon strok değerini veren $\left(x_{1}-x_{2}\right)$ ve yolcu konforunu etkileyen parametre olan araç gövde ivmesi $\left(d^{2} x_{1} / d t^{2}\right)$ verileri incelenmiştir.

ISO (International Organization for Standardization) 8608'e göre yol kalitesi A ile $\mathrm{H}$ arasında sınıflanmıştır. A en kaliteli yolu $\mathrm{H}$ ise en kötü yolu ifade etmektedir. Bu çalışmada $\mathrm{C}$ sınıfı bir yol girişi modele uygulanmıştır (Şekil 4). Araç hızı ise $15 \mathrm{~m} / \mathrm{s}$ olarak kabul edilmiștir.

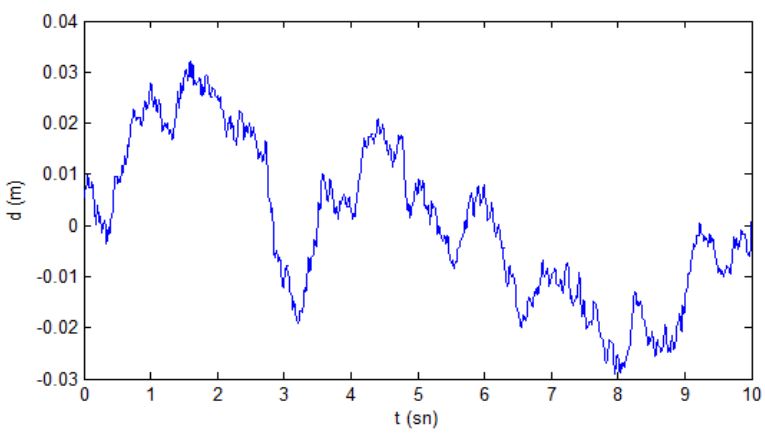

Şekil 4. ISO C sınıfı yol girişi.

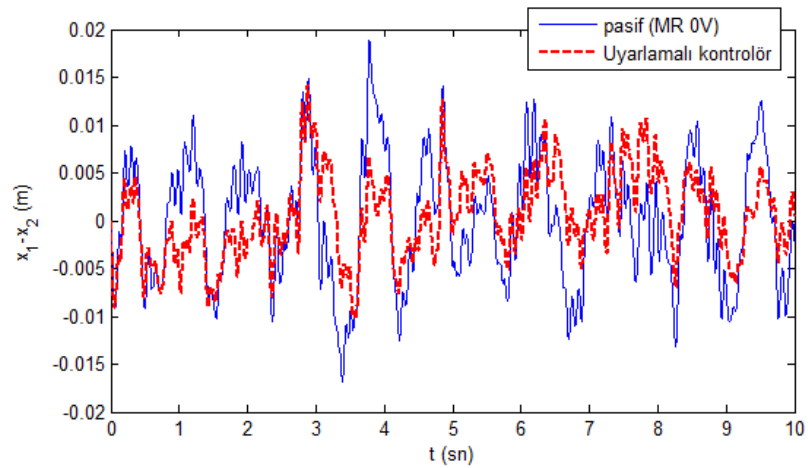

Şekil 5. Süspansiyon yer değiştirmesi.

Elde edilen grafiklerde düz çizgi MR dampere herhangi bir gerilim uygulanmadığı durumu (pasif), kesikli çizgi ise yol girişinin uyarlamalı kontrolör ile kontrol edilen durumunu ifade etmektedir. Şekil 5'te süspansiyon rölatif yer değiştirmesi sonucu verilmiştir. Klasik bir MR damperin strok değeri $74 \mathrm{~mm}$ (RD 8041-1) olarak bilinmektedir. Dolayısıyla Şekil 5 incelendiğinde, hem pasif duruma ait cevapların hem de uyarlamalı kontrol durumuna ait cevaplarının süspansiyon strok limitlerini aşmadığı görülmektedir. 


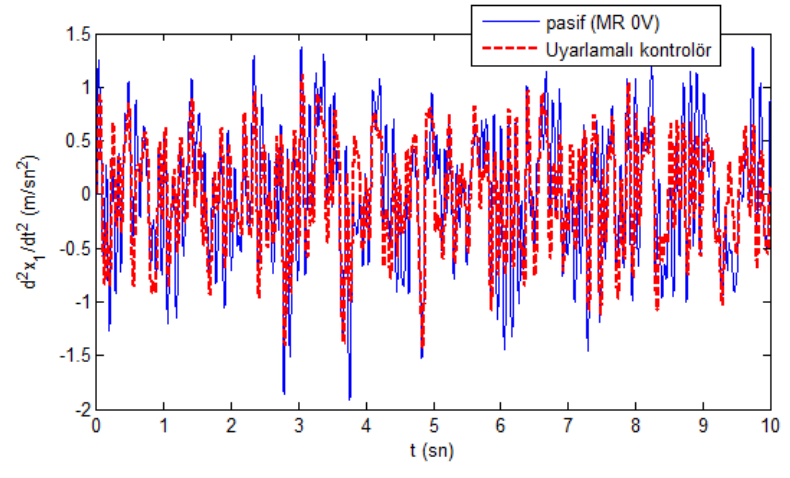

Şekil 6. Taşıt gövdesinin ivmesi.

Şekil 6'da taşıt gövdesine ait ivme cevapları verilmiştir. Konfor açısından en önemli veri ivme cevaplarıdır. Şekil 6'da uyarlamalı kontrolörün taşıt gövdesine ait ivmeleri pasif duruma göre bastırdığı açıkça görülmektedir.

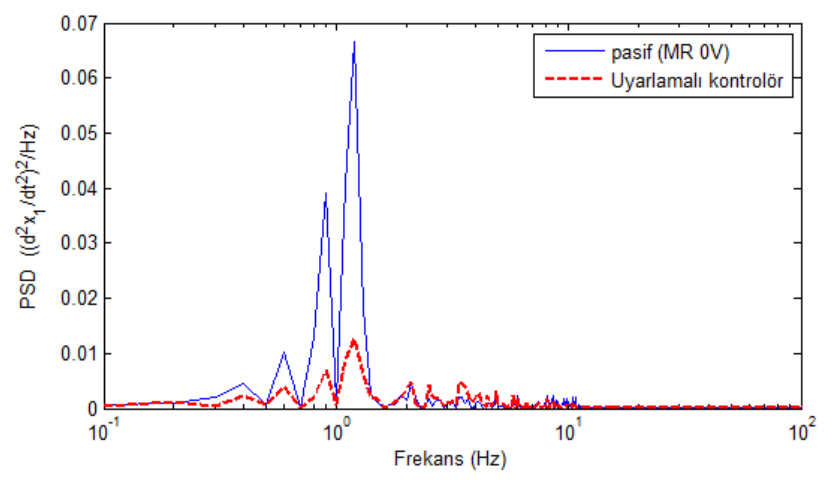

Şekil 7. Taşıt gövdesinin ivmesinin PSD cevabı.

Şekil 7'de frekans alanında analiz yapmak için taşıt gövdesine ait ivme değerinin PSD (Power Spectral Density) cevapları gösterilmiştir. Şekil 7 incelendiğinde, önerilen kontrolörün taşıt gövdesinin doğal frekansında oluşan rezonans tepelerini önemli olçüde bastırdığı görülmektedir.

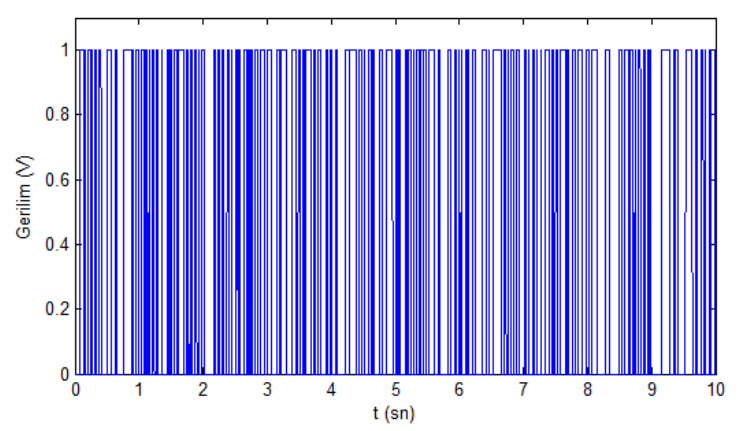

Şekil 8. MR dampere uygulanan gerilim.

Şekil 8'de MR dampere kontrolör tarafindan gönderilen gerilim miktarı verilmiştir. MR dampere uygulanan gerilim 1 Volt ile sınırlandırılmıştır. Böylece, kontrol işlemi için düşük enerji gereksinimi sağlanarak titreşimler bastırılmıştır.

Sonuçları sayısal olarak değerlendirmek için cevapların RMS (Root Mean Square) ortalamaları alınmıştır.
Grafiklerde karmaşıklığı önlemek amacıyla sadece yol girişinin ölçülmediği durum için tasarlanan kontrolör cevapları verilmiştir. RMS ortalamalarında ise hem yol girişinin ölçüldüğü hem de yol girişinin ölçülmediği durumlar karşılaştırılmıştır. Tablo 3 incelendiğinde, yol girişinin ölçüldüğü kabulüyle tasarlanan uyarlamalı kontrolör, pasif duruma göre taşıt gövdesinin yer değiştirmesinin RMS ortalaması açısından \%14 iyileşme sağlamıştır. Önerilen, yol girişinin ölçülmediği durum için tasarlanan uyarlamalı kontrolör ise \%13 oranında iyileşme sağlamıştır. Taşıt gövdesinin ivmesinin RMS ortalaması açısından bu çalışmada önerilen, yol girişinin ölçülmediği durum için tasarlanan uyarlamalı kontrolör \%30 iyileşme sağlarken, yol girişinin ölçüldüğü uyarlamalı kontrolör ise \%31 iyileşme sağlamıştır.

Tablo 3. RMS ortalamaları

\begin{tabular}{|c|c|c|}
\hline Durum & $x_{1}$ & $\ddot{x}_{1}$ \\
\hline Pasif & 0.0170 & 0.6013 \\
Uyarlamalı & 0.0148 & 0.4173 \\
(yol girişi ölçülmüyor) & & \\
Uyarlamalı & 0.0146 & 0.4149 \\
(yol girişi ölçülüyor) & & \\
\hline
\end{tabular}

\section{DEĞERLENDIRME VE SONUÇ}

Bu çalışmada, çeyrek taşıt titreşimlerini bastırmak için yarı aktif bir eleman olan MR damper kullanılmıştır. Yol girişinin ölçülmesindeki güçlükler göz önüne alınarak, bu girişin ölçülmesine ihtiyaç duymayan lineer olamayan bir kontrolör önerilmiştir. Tasarlanan kontrolcünün etkinliği MATLABSimulink programı ile yapılan simülasyon sonuçları ile zaman ve frekans açısından incelenmiştir. Grafiklerde karmaşıklığı önlemek için sadece önerilen yöntemin cevapları pasif cevaplarla karşılaştırılmıştır. Ayrıca, önerilen yöntemin RMS değerleri, yol girişinin ölçüldüğü kabulüyle elde edilen RMS sonuçları ile karşılaştırılmıştır. Yol girişinin ölçüldüğü durumda tasarlanan uyarlamalı kontrolcü ile önerilen yöntemin sonuçlarının birbirine çok yakın olduğu görülmüştür (\%1). Dolayısıyla bu yöntem ile, yol girişin ölçülmesine ihtiyaç duyulmadan taşıt titreşimlerinin bastırılabileceği ve ölçüm maliyetlerinin ortadan kaldırılarak, daha ekonomik bir yapı ile benzer kontrol performansının elde edilebileceği gösterilmiştir.

\section{KAYNAKÇA}

[1] M. Paksoy, R. Guclu, and S. Cetin, "Semiactive Self-Tuning Fuzzy Logic Control of Full Vehicle Model with MR Damper," Advances in Mechanical Engineering, vol. 6, p. 816813, 2014.

[2] X. Tang, H. Du, S. Sun, D. Ning, Z. Xing, and W. Li, "Takagi-Sugeno Fuzzy Control for Semi-Active Vehicle Suspension With a Magnetorheological Damper and Experimental Validation," IEEE/ASME Transactions on Mechatronics, vol. 22, no. 1, pp. 291-300, 2017. 
[3] M. Yu, X. Dong, S. Choi, and C. Liao, "Human simulated intelligent control of vehicle suspension system with MR dampers," Journal of Sound and Vibration, vol. 319, no. 3-5, pp. 753-767, 2009.

[4] G. Kararsiz, M. Paksoy, M. Metin, and H. I. Basturk, "Hardware-In-the-Loop Simulation for SemiActive Suspension System with Using Adaptive Backstepping Approach," 2018 6th International Conference on Control Engineering \& Information Technology (CEIT), 2018.

[5] S. Cetin, E. Zergeroglu, S. Sivrioglu, and I. Yuksek, "A new semiactive nonlinear adaptive controller for structures using MR damper: Design and experimental validation," Nonlinear Dynamics, vol. 66, no. 4, pp. 731743, 2011.

[6] H. Metered, P. Bonello, and S. Oyadiji, "Nonparametric Identification Modeling of Magnetorheological Damper Using Chebyshev Polynomials Fits," SAE International Journal of Passenger Cars Mechanical Systems, vol. 2, no. 1, pp. 1125-1135, 2009.

[7] D. H. Wang and W. H. Liao, "Modeling and control of magnetorheological fluid dampers using neural networks," Smart Materials and Structures, vol. 14, no. 1, pp. 111-126, Aug. 2004.

[8] J. C. Tudón-Martínez and R. Morales-Menendez, "Nonparametric Modeling of an Automotive Damper Based on ANN: Effect in the Control of a Semi-active
Suspension," Studies in Computational Intelligence Computational Intelligence, pp. 295-309, 2015.

[9] C. Sakai, H. Ohmori, and A. Sano, "Modeling of MR damper with hysteresis for adaptive vibration control," 42nd IEEE International Conference on Decision and Control (IEEE Cat. No.03CH37475).

[10] A. S. Yildiz, S. Sivrioglu, E. Zergeroglu, and S. Cetin, "Adaptive control of semiactive quarter car model with MR damper," 2013 9th Asian Control Conference (ASCC), 2013.

[11] K. M. Mahala, P. Gadkari, and A. Deb, "Mathematical models for designing vehicles for ride comfort," 2009 2nd International Conference on Research into Design (ICORD 09), Bangalore, India 2009.

[12] G. Kararsız and H. Baştürk, "Aktif süspansiyon sistemleri için bilinmeyen bozucu etkisi altında uyarlamalı kontrolcü tasarımı," Pamukkale Üniversitesi Mühendislik Bilimleri Dergisi, vol. 24, pp. 1403-1408, 2018.

[13] H. I. Baştürk and M. Krstic, “Adaptive Cancellation of Matched Unknown Sinusoidal Disturbances for LTI Systems by State Derivative Feedback," Journal of Dynamic Systems, Measurement, and Control, vol. 135, no. 1, 2012. [14] V. O. Nikiforov, "Observers of External Deterministic Disturbances. I. Objects with Known Parameters," Automation and Remote Control, vol. 65, no. 10, pp. 1531-1541, 2004. 